\title{
Statistical considerations on the extinction error variance for the Raman lidar inversion algorithm
}

\author{
Francesc Rocadenbosch, Adolfo Comerón, Michaël Sicard, Mohd Nadzri Md Reba \\ Universitat Politècnica de Catalunya (UPC), Dep. of Signal Theory and Communications (TSC), \\ Remote Sensing Lab (RSLAB), C/Jordi Girona, 1-3, D4-016, \\ 08034 Barcelona, SPAIN. \\ roca@tsc.upc.edu
}

\begin{abstract}
At the basis of the Raman lidar extinction inversion algorithm is the derivative of the logarithm of the ratio between the atmospheric nitrogen number density and the rangecorrected Raman power return. While its computation is straightforward under ideal (noiseless) conditions, this is not the case under low signal-to-noise ratios, for which the observation noise may lead the logarithm to singular values. This work presents an analytical-statistical overview of the inversion problem and related errorbars, tentative noise limiting criteria, and justifies the approximations at play to estimate the inversion error by means of error-propagation techniques for high signalto-noise ratios. Simulation examples consider a 532/607-nm elastic-Raman system.
\end{abstract}

Keywords- Lidar, Raman inversion, extinction, errorbars, logtransformed noise.

\section{INTRODUCTION}

Combination of, at least, one elastic and one Raman lidar channel for independent inversion of the atmospheric optical parameters, namely, extinction and backscatter is becoming common practice in the international community and, particularly, in EARLINET (European AeRosol LIdar NETwork) [1][2][3][4].

Ansmann's et al. Raman lidar algorithm [5] provides analytical solution of the inversion problem, and standard error propagation techniques are used to derive (aerosol) extinction, backscatter and lidar-ratio errorbars [6]. At the basis of this algorithm is the derivative of the logarithm of the ratio between the atmospheric nitrogen number density and the rangecorrected observable (i.e., noise-corrupted) Raman power return. Computation of this kernel is not straightforward for, at least, three main reasons: 1) There is an increasing statistical likelihood for progressively decreasing signal-to-noise ratios that observation noise causes singular exceptions in the logarithm. This forces inclusion of appropriate decision rules such as resetting negatives values of the logarithm argument to some limiting threshold or others. 2) Assuming the simplification that errors on the nitrogen number density can be neglected, noise at the logarithm output cannot "a priori" be assumed Gaussian. In the most general case, it is just logtransformed noise, which emerges as a non-symmetric noise in amplitude over the logarithm curve (the so-called S-curve next) for low signal-to-noise ratios. In contrast, 3) standard error propagation techniques, which are widely used in the lidar community, are inherently perturbational, which means that errors on an input variable are directly translated to the output variable simply scaled by (the modulus of) a partial derivative. Formally, this assumes the questionable hypothesis that noise over the S-curve still retains Gaussian statistics.

In addition to the reasons stated above, the derivative operator tends to magnify noise over the S-curve. Though different noise-reduction approaches are feasible, here we consider the case in which the derivative is computed by estimating the slope along successive straight-line fits over the S-curve.

All along this work, we assume the additional oversimplifying hypotheses that the statistical error due to signal detection is the dominant error source and that "calibrated" (i.e., background subtracted) lidar signals are corrupted by Gaussian observation noise. Systematic errors such as those due to e.g., the estimation of temperature/pressure, ozone profiles, Angström coefficient or multiple scattering are not considered (superposition principle for independent error sources).

This paper is organised as follows: In Sect. II, we review the Raman lidar inversion algorithm and present a generalized formulation of the noise-induced extinction errorbar variance. In Sect. III, noise threshold-limiting rules are presented (point (1) above). The estimated error variance is compared with that from classic error-propagation techniques [6] (points (2)-(3) above). Concluding remarks are given in Sect. IV.

\section{RAMAN LIDAR INVERION AND ERROR ASSESSMENT}

\section{A. Review of the Raman inversion algorithm}

We depart from the well-known Ansmann's et al. elasticRaman lidar algorithm (adapted from [7]), to independently derive the aerosol extinction at emission wavelength, $\lambda_{0}$,

$$
\alpha_{\lambda_{0}}^{a e r}(R)=\frac{\frac{d}{d R}\left[\ln \frac{N_{R}(R)}{R^{2} P_{\lambda_{R}}(R)}\right]-f(R)}{g(R)},
$$

with

$$
f(R)=\alpha_{\lambda_{0}}^{m o l}(R)+\alpha_{\lambda_{R}}^{m o l}(R), \quad g(R)=1+\left(\frac{\lambda_{0}}{\lambda_{R}}\right)^{\kappa},
$$


where $P_{\lambda_{R}}(R)$ is the Raman return power from range $R, \lambda_{0}$ and $\lambda_{\mathrm{R}}$ are, respectively, the emission and reception (Raman) wavelengths, $N_{R}(R)$ is the $N_{2}$ molecule number density, mol and aer are reminders of "molecular" and "aerosol" components, respectively, and $\kappa$ expresses the $\lambda^{-\kappa}$ particle scattering wavelength dependence.

So as to compute the logarithm kernel of (1), $\ln \left[\frac{N_{R}(R)}{R^{2} P_{\lambda_{R}}(R)}\right]$, -the so-called S-curve next-, a sliding-window linear fitting procedure of $\mathrm{N}$ samples [1] is applied around each successive sample (formally, fitting intervals are defined as $I_{j}=\left[R_{j}, R_{j+N-1}\right]$, $j=1,2, \ldots, P-N+1$, with $P$ the number of samples). This yields a much less noisy S-curve with inversion resolution, $\Delta R_{f}=(N-1) \Delta R$, where $\Delta R$ is the raw resolution. It is also assumed that any spatial pre-processing smoothing gain is assimilated into an equivalent input signal-to-noise ratio (SNR).

Figs. 1-2 reproduce a 532/607-nm elastic/Raman inversion example in the 0.2 -to- $4 \mathrm{~km}$ range $(\Delta R=7.5 \mathrm{~m})$ with a simulated boundary layer ending at $R=3 \mathrm{~km}$. Simulated noise-corrupted Raman returns have been generated from the input aerosol extinction profile of Fig. 1 (mean extinction, $\overline{\alpha_{\lambda_{0}}^{a e r}}=1 \mathrm{~km}^{-1}$ in the 0.2-to-3 km range), a US-standard atmosphere model [8] (molecular components) with ground level conditions, $\mathrm{T}_{0}=-2$ $\left.{ }^{\circ} \mathrm{C}, \mathrm{P}_{0}=1025 \mathrm{hPa}\right), \kappa=1.8$, and a SNR at the starting range, $\operatorname{SNR}\left(R_{\text {min }}\right)=500$ (see Fig. 2). The count rate is high enough (> 10 counts/s) so as to assimilate Poisson statistics into equivalent Gaussian ones. The fitting length (inversion resolution) used is $120 \mathrm{~m}$ up to $1 \mathrm{~km}, 180 \mathrm{~m}$ up to $3 \mathrm{~km}$, and $375 \mathrm{~m}$ up to $4 \mathrm{~km}(N=17,25$, and 51 samples, respectively).

Fig. $2 \mathrm{~b}$ depicts numerical exception events (i.e. noise spikes causing negative values in the logarithm argument) as function of range due to the low SNR in the $2-4 \mathrm{~km}$ range.

\section{B. Formulation of the noise-induced extinction errorbars}

Noise-induced inversion errors can easily be evidenced by rewriting the log kernel of (1) as

$$
S_{i}=\ln \left[\frac{N_{R, i}}{R_{i}^{2}\left(P_{i}+n_{i}\right)}\right]=-\ln \left(R_{i}^{2} \frac{P_{i}}{N_{R, i}}\right)-\ln \left(1+\frac{n_{i}}{P_{i}}\right),
$$

where subindex "i" is a reminder of sampling at observation cell $R=R_{i}$, and we have substituted the Raman power, $P_{\lambda_{R}}(R)$, by the superposition of its signal (mean photocount) and (equivalent zero-mean Gaussian) noise components, $P_{i}$ and $n_{i}$, respectively.

Let $s_{j}(R)=\hat{m}_{j} R+\hat{c}_{j}$ be the straight line fit to an interval $I_{j}$ of the observable (i.e., noise-corrupted) S-curve (3), and $s_{j}(R)=m_{j} R+c_{j}$ that to the ideal (noiseless) S-curve. At the same time, let's assume that curvature errors (i.e., the error arising when trying to fit a straight line to a curve) are negligible in front of noise-induced errors. Under these circumstances, the inverted aerosol extinction becomes

$$
\hat{\alpha}_{\lambda_{0}, j}^{\text {aer }}(R)=\frac{\hat{m}_{j}-f(R)}{g(R)} .
$$

Note that (4) is just a stochastic estimate (slope $\hat{m}_{j}$ and ordinate intercept $\hat{c}_{j}$ change at each successive noise realization) of the "true" deterministic extinction solution $\left(n_{i}=0\right.$ in (3))

$$
\alpha_{\lambda_{0}, j}^{a e r}(R)=\frac{m_{j}-f(R)}{g(R)} .
$$

From (4)-(5) above, the error estimation variance can readily be computed as

$$
\operatorname{Var}\left[\varepsilon_{\alpha_{j}}\right]=\operatorname{Var}\left[\left(\hat{\alpha}_{j}-\alpha_{j}\right)\right]=\frac{\operatorname{Var}\left[\hat{m}_{j}\right]}{g^{2}} .
$$

If, in (3), we define the log-transformed noise as

$$
\Omega_{i}=\ln \left(1+\frac{N_{i}}{P_{i}}\right)
$$

(upper case letters are used for random variables and lower case for their numerical values) then the variance on the estimated slope can be computed as [9][10]

$$
\operatorname{Var}\left(\hat{m}_{j}\right)=\frac{1}{N^{2} Q^{2}} \sum_{i=1}^{N}\left(R_{i}-\bar{R}\right)^{2} \operatorname{Var}\left[\Omega_{i}\right], \quad Q=\frac{1}{N} \sum_{i=1}^{N}\left(R_{i}^{2}\right)-\bar{R}^{2} .
$$

Equations (6) and (8) are most general expressions to compute aerosol extinction errorbars.

\section{STATISTICAL CONSIDERATIONS}

\section{A. Statistics of the log-transformed noise and threshold limiting conditions}

As long as we move to the far range (Fig. 3a), where the SNR is poorer, log-transformed noise $\Omega_{i}$ progressively exhibits a non-symmetric, thus non-Gaussian behaviour. Moreover, there is an increasing likelihood for noise spikes at a range $R=R_{i}$ to grow larger and larger so that $n_{i} \leq-P_{i}$. This causes a singularity (see (3), (7) and Fig.2b).

Though research is under way, bi-lateral threshold limiting (Fig. 4), i.e., limiting both positive and negative spikes of $n_{i}$ to some threshold $\pm\left|n_{l, i}\right|$ ensuring $n_{i} / P_{i}>-1$, prior to computing the logarithm of the S-curve, is a convenient way. This is illustrated in Fig. $3 b$ (note that discontinuities in the dashed grey-circled trace of Fig. $3 \mathrm{~b}$ indicate $(\mathrm{NaN})$ singularities). A similar methodology applied to slope-method processing of elastic signals can be found in [10].

Two different threshold setting criteria have been studied: 1) $\eta$-limiting threshold,

$$
\left|n_{l, i}\right|=\eta \sigma_{i}
$$

which means setting the threshold at e.g., the 1-,2-,3- $\sigma$ level $(\eta=1,2,3)$ of observation noise, $n_{i}$. And, 2) $\gamma$-limiting threshold, where 


$$
\gamma=\left|n_{l, i}\right| / P_{i}
$$

In this case, the threshold $n_{l, i}$ is directly related to (7) and limits the range-dependent SNR to a floor, $S N R_{\lim }=1 / \gamma$. Fig. 5 plots numerical computation of $\operatorname{Var}\left[\Omega_{i}\right]$ (cases $\eta=1,2,3, \gamma=0.1$ ), after which it is straightforward to compute (8) and the soughtafter extinction errorbars (6).

\section{B. The perturbational vs. the statistical approach}

Whiteman [6] provides an excellent summary of classic error-propagation techniques applied to assess the inversion error variance for different fitting methods. In the case of linear fitting and disregarding errors on the nitrogen number density, the log-transformed noise variance, $\operatorname{Var}\left[\Omega_{i}\right]$, is computed as (p. 3362),

$$
\sigma_{g(z)}^{2}=\sigma_{P(z)^{2}} / P(z)^{2} \rightarrow \operatorname{Var}\left[\Omega_{i}\right]=1 / \operatorname{SNR}(R)^{2},
$$

(The right part of (11) translates referenced source notation into our notation).

When (11) is cross-examined with the more general statistical formulation of (7) and Sects. II-III, it emerges that (11) represents just the perturbational approximation, $x=n_{i} / P_{i}<<1$ in (7), (equivalently $\mathrm{SNR}>>1$ in the fitting interval $\mathrm{I}_{\mathrm{j}}$ ), so that $\ln (1+x) \approx x$. Thus, the $\log$-transformed noise $\Omega_{i}$ becomes $\Omega_{i} \approx N_{i} / P_{i}$, and the Gaussian behavior is still retained. Formally, the condition $\mathrm{SNR}>>1$ implies that any reasonable limiting threshold is far from activating and thus has virtually no effect on the Gaussian p.d.f. Quantitatively, Fig. 5 illustrates that (11) perturbational variance starts to depart from e.g., the $\gamma$-limited statistical variance ((7),(10)) for SNRs as low as $\mathrm{SNR}=3(+8.5 \%$ relative error $)$ in front of $\mathrm{SNR}=10(-2.6 \%)$.

Finally, Fig. 6 compares aerosol extinction errorbars $((6),(8))$ when computed from both the perturbational and the statistical approaches with a Monte Carlo's method, which is used as reference. In Monte Carlo's method a set of 100 different noise-corrupted Raman returns are inverted via (1) (and noise limited accordingly). Errorbars are computed from the upper and lower envelopes of the 100 inverted extinction profiles. Both the perturbational and the statistical approaches yield similar errorbars (relative error $10 \%$ ) for ranges $\mathrm{R}<1.6$ $\mathrm{km}$, where $\mathrm{SNR}>10$. In contrast, for moderate $\mathrm{SNRs}(\mathrm{SNR} \approx 10$, $\mathrm{R} \approx 1.8 \mathrm{~km}$ ), the perturbational method overestimates the errorbars by some $40 \%$ and up to one order of magnitude at $\mathrm{R} \approx 3 \mathrm{~km}$, where the SNR approaches unity.

\section{CONCLUSION}

A statistical formulation of the noise-induced aerosol extinction errorbars associated to the Raman lidar algorithm has been presented when a linear fit is performed over the Scurve. The inversion error variance is directly related to the fitting/sampling parameters used and to the variance of the logtransformed noise.

Classic error-propagation techniques, which are perturbational in nature, are of valid application and thus convergent to results provided by the more general statistical formulation presented for high SNRs (tentatively $\mathrm{SNR} \geq 10$ ) virtually irrespective of the limiting rule chosen. For lower SNRs threshold-limiting rules are needed to avoid singularities in the S-curve as well as a formulation of the p.d.f., which is no longer Gaussian.

\section{ACKNOWLEDGMENT}

The authors wish to acknowledge the following entities for partially supporting this research work and lidar systems developed at UPC: European Union and FEDER funds under the EARLINET-ASOS (EU Coordination Action) contract $\mathrm{n}^{\circ}$ 025991 (RICA), and (EU Specific Support Action) contract $n^{\circ}$ 011863 (RIDS): "Technology development programme towards a European Extremely Large Telescope"; MCYT (Spanish Ministry of Science and Technology) and FEDER funds under the projects TEC2006-07850/TCM and REN200309753-C02-02, Complementary Actions CGL2006-26149E/CLI, CTM2006-27154-E/TECNO, and Special Action REN2002-12784-E; MITYC (Spanish Ministry of Industry, Tourism and Commerce) under the PROFIT project, CIT020400-2005-56. MCYT is also thanked for the Ramón y Cajal position hold by Dr. M. Sicard, and Local Government of Catalonia (Generalitat de Catalunya/AGAUR) for Mr. Md. Reba's predoctoral fellowship.

\section{REFERENCES}

[1] I. Mattis, A. Ansmann, D. Müller, U. Wandinger, D. Althausen, "Multiyear aerosol observations with dual-wavelength Raman lidar in the framework of EARLINET," J. Geophys. Res., 109, 2004JD004600, (2004).

[2] D.N. Whiteman, "Application of statistical methods to the determination of slope in lidar data," Appl. Opt. 38(15), 3360-3369 (1999).

[3] A. Ansmann, M. Riebesell, and C. Weitkamp, "Measurement of atmospheric aerosol extinction profiles with a Raman lidar", Opt. Lett. 15, 746-748, 1990.

[4] A. Ansmann, U. Wandinger, M. Riebesell, C. Weitkamp, W. Michaelis, "Independent measurement of extinction and backscatter profiles in cirrus clouds by using a combined Raman elastic-backscatter lidar," Appl. Opt. 31(33), 7113-7131 (1992).

[5] G. Pappalardo, A. Amodeo, M. Pandolfi, U. Wandinger, A. Ansmann, J. Bösenberg, V. Matthias, V. Amiridis, F. De Tomasi, M. Frioud, M. Iarlori, L. Komguem, A. Papayannis, F. Rocadenbosch, X. Wang. "Aerosol lidar intercomparison in the framework of the EARLINET project. 3. Raman lidar algorithm for aerosol extinction, backscatter and lidar ratio." Appl. Opt., 43 (28), 5370-5385 (2004).

[6] U. Wandinger, I. Mattis, M. Tesche, A. Ansmann, J. Bösenberg, A. Chaikovski, V. Freudenthaler, L. Komguem, H. Linné, V. Matthias, J. Pelon, L. Sauvage, P. Sobolewski, G. Vaughan, M. Wiegner, "Air-mass modification over Europe: EARLINET aerosol observations from Wales to Belarus," J. Geophys. Res., 109, 2004JD005142, (2004).

[7] V. Matthias, D. Balis, J. Bösenberg, R. Eixmann, M. Iarlori, L. Komguem, I. Mattis, A. Papayannis, G. Pappalardo, M.R. Perrone, X. Wang, "The vertical aerosol distribution over Europe: Statistical analysis of Raman lidar data from 10 EARLINET stations," J. Geophys. Res., 109, 2004JD004638, (2004).

[8] B.A. Bodhaine, N.B. Wood, E.G. Dutton, J.R. Slusser, "On Rayleigh Optical Depth Calculations," J. Atmospheric and Oceanic Technology 16(11), 1854-1861 (1999).

[9] R.J. Barlow, "Least Squares", Chap.6 in Statistics: A Guide To The Use Of Statistical Methods In The Physical Sciences, F. Mandl, R.J. Ellison, D.J. Sandiford, Eds., (Wiley, Chichester, England, 1989).

[10] F. Rocadenbosch, A. Comerón, L. Albiol, "Statistics Of The SlopeMethod Estimator," Appl. Opt. 39(33), 6049-6057 (2000). 


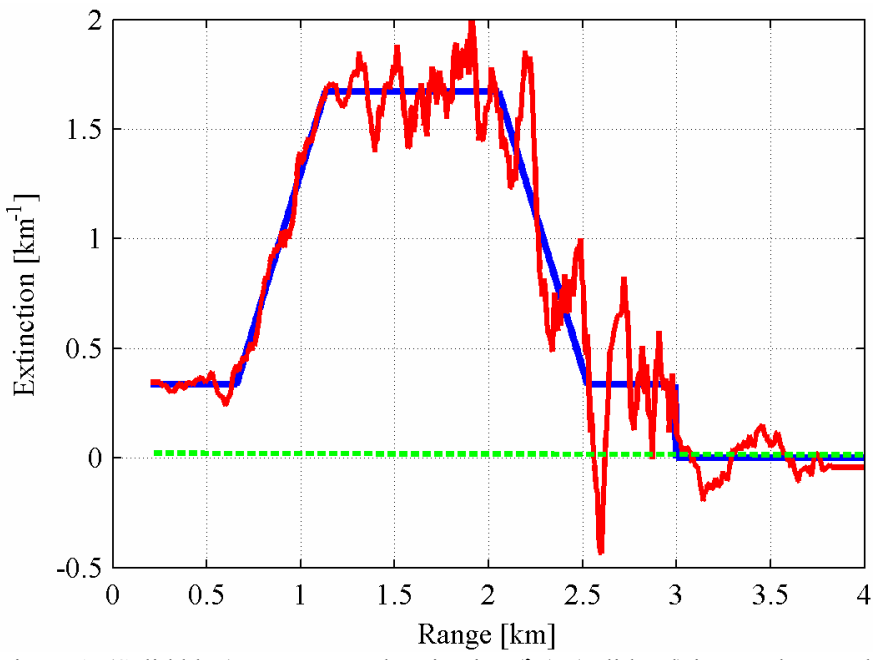

Figure 1. (Solid blue) Input aerosol extinction $\left(\lambda_{0}\right)$, (solid red) inverted aerosol extinction, (dashed green) total molecular extinction, $\mathrm{f}(\mathrm{R})$.

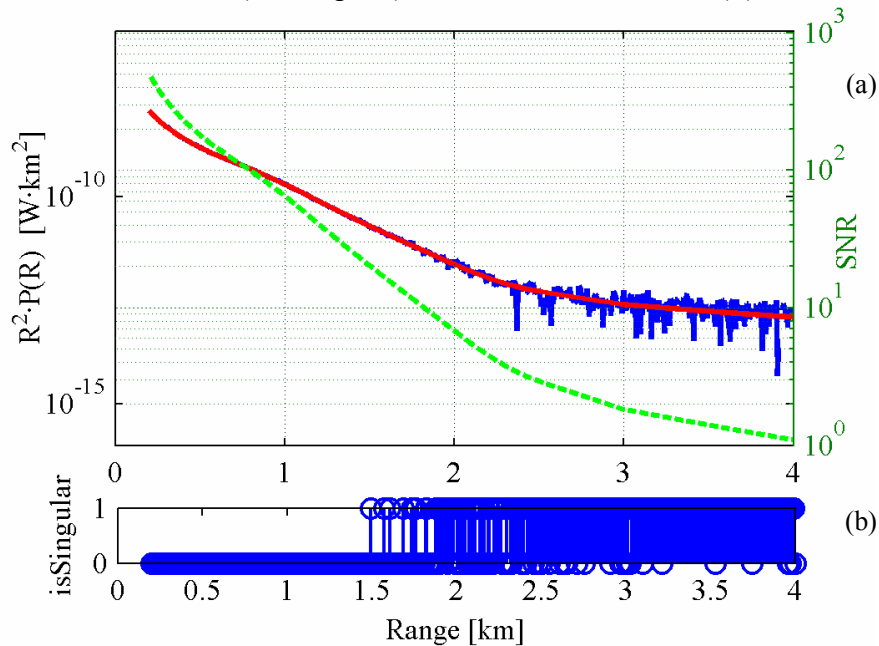

Figure 2. (a) (left) Superimposed range-corrected noisy observable/noiseless power, $R^{2} z(R)$ and $R^{2} P(R)$ (solid blue/red), respectively, along with the SNR (right). (b) Associated singularities when computing the logarithm (S-curve).
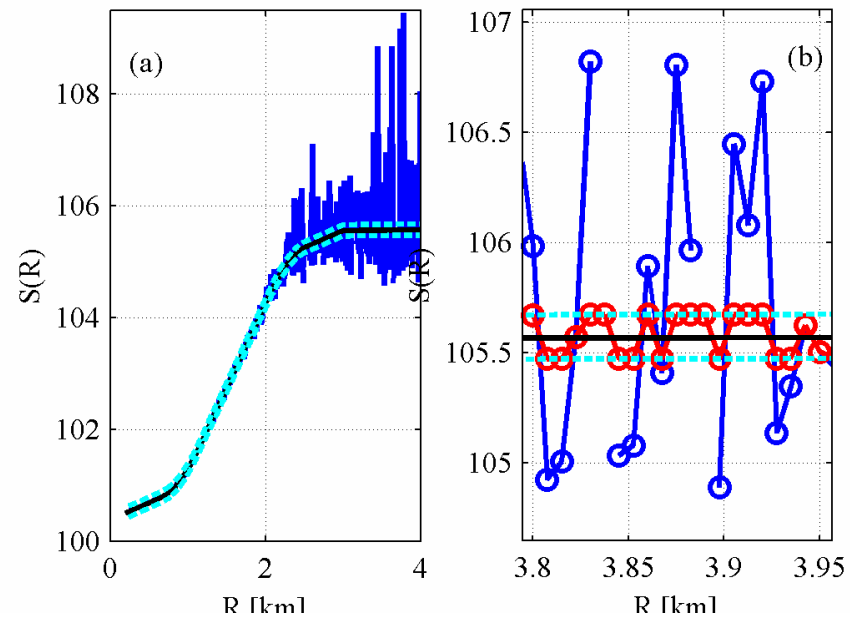

Figure 3. Threshold limiting $(\gamma=0.1)$. (a) Noisy S-curve (solid-blue) and threshold limited one (solid-black). (b) Detail of (a): (blue/red circles) Noisy/threshold-limited samples, (dashed cyan) threshold, (solid black) noiseless S-curve.

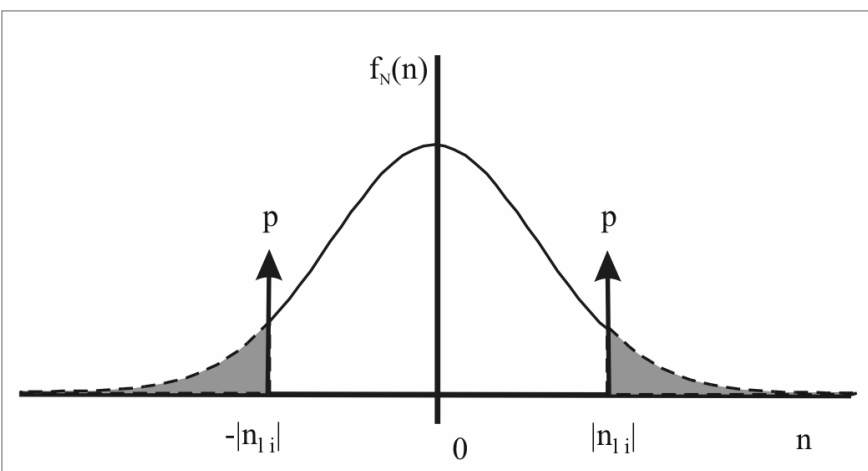

shaded area $=\mathrm{p}$

Figure 4. Probability density function (p.d.f.) of the bilateral threshold-limited Gaussian random variable $\mathrm{N}_{\mathrm{i}}$.

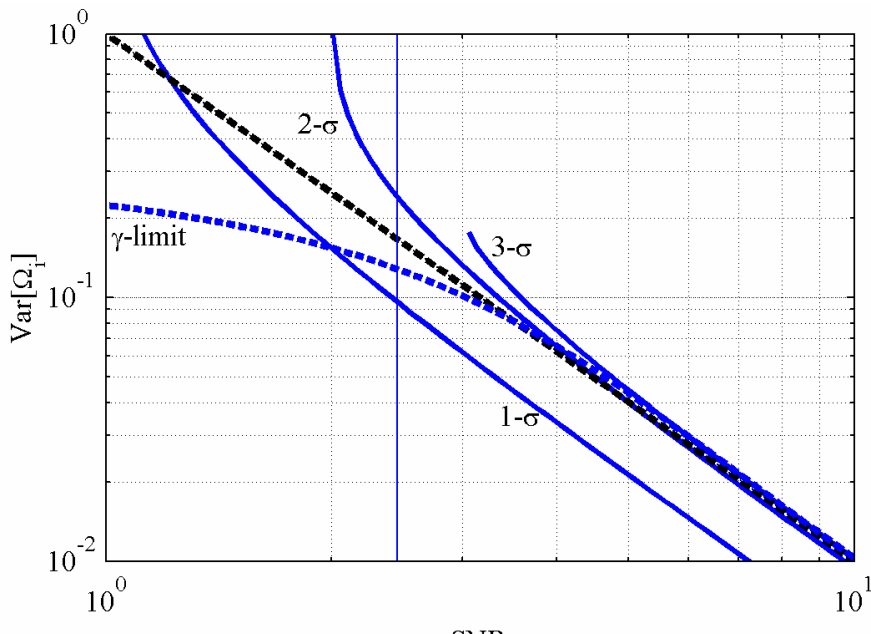

SNR

Figure 5. Variance of log-transformed noise, $\Omega_{\mathrm{i}}$. (Dashed black) perturbational approach (no limiting threshold), (solid blue) 1-, 2-, and 3- $\sigma \eta$-limiting

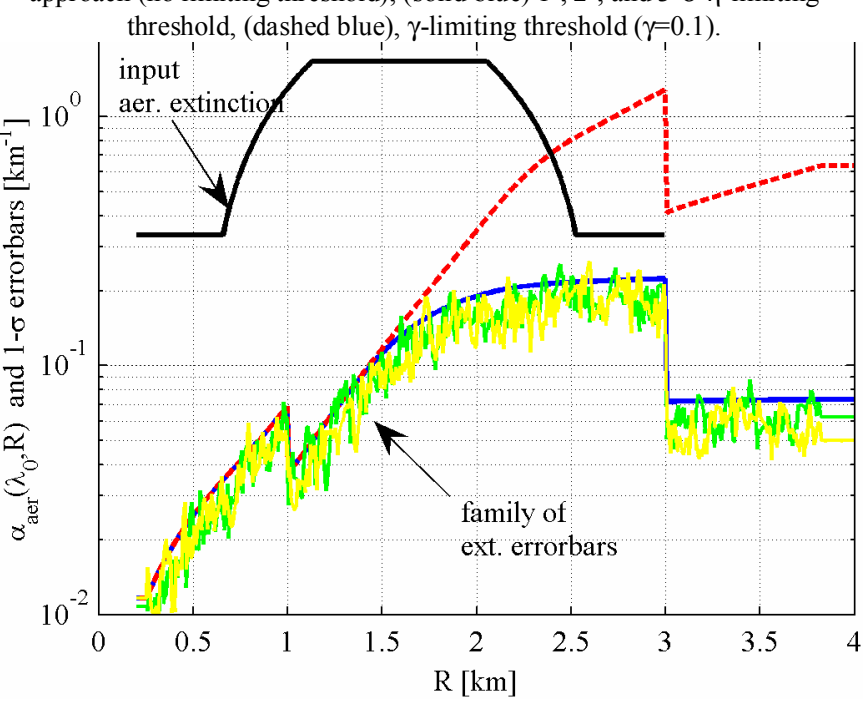

Figure 6. Comparison among different inversion errorbars: (solid blue) statistical ones, (noisy green/yellow) Monte-Carlos', (dashed red) perturbational ones. Input aerosol extinction profile (Fig. 1) shown for comparison. 